\title{
Wachstum deutlich stärker als der Markt
}

Das Jahr 2015 war für CAMLOG ein weiteres Rekordjahr. Der Implantathersteller verzeichnete in Deutschland ein knapp zweistelliges Umsatzwachstum und übertrifft damit das Marktwachstum, das auf 2-3\% geschätzt wird. „Wir sind mit der Entwicklung im vergangenen Jahr hochzufrieden. Jedes 4. Zahnimplantat kommt

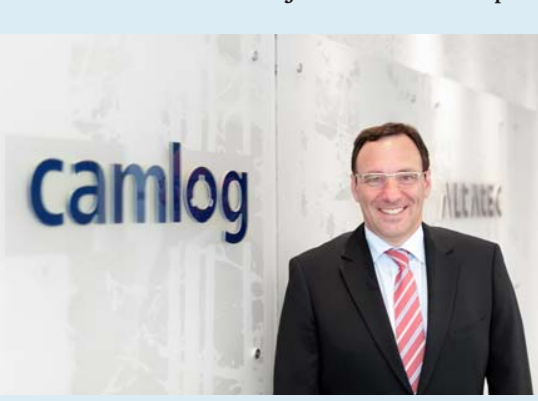

in

Deutschland von CAMLOG. Unsere $\mathrm{Ge}$ schäftspolitik der Kontinuität und Ver-

lässlichkeit sowie unsere innovativen Produktlösungen und Konzepte werden sehr geschätzt. Darauf bauen wir auch 2016 und bieten unseren Kunden neben einer hervorragenden Produktqualität ein partnerschaftliches Angebot zum beiderseitigen Nutzen“, resümiert Michael Ludwig, Geschäftsführer der CAMLOG Vertriebs GmbH.2013 hatte CAMLOG bei der Internationalen Dental-Schau in Köln mit großem Aufsehen das iSy Implantatsystem eingeführt und sich damit ein 2. Standbein im viel beachteten Value-Segment geschaffen. Ludwig: „Mit iSy liegen wir goldrichtig. Kunden unserer Premium-Mitbewerber suchen bewusst nach einem kostengünstigen Zweitsystem von einem renommierten Hersteller, für den die Qualität der Produkte an 1. Stelle steht. Das iSy System sichert uns heute schon einen großen Teil des Neukundengeschäfts. Dieser Trend wird sich weiter fortsetzen, zumal iSy auch optimal auf DEDICAM - und damit ein weiteres wichtiges Zukunftssegment - abgestimmt ist.“ DEDICAM steht bei CAMLOG für digitale Prothetiklösungen und konnte seinen Umsatz nach Unternehmensangaben zum 2. Mal in Folge verdoppeln. CAMLOG etabliert sich damit auch auf dem CAD/CAM-Markt durch ein maßgeschneidertes Angebot an Scan-, Design- und Fertigungsdienstleistungen basierend auf offenen Schnittstellen.Weitere Informationen zu dem Produktangebot, den Serviceleistungen, Fortbildungsveranstaltungen und vielem mehr von CAMLOG bekommen Sie auf www.camlog.de oder telefonisch unter 07044 9445-100.

Nach einer Pressemitteilung der CAMLOG Vertriebs GmbH, Wimsheim 